\title{
Open the Way to Future Networks - A Viewpoint Framework from ITU-T*
}

\author{
Daisuke Matsubara $^{1}$, Takashi Egawa ${ }^{2}$, Nozomu Nishinaga ${ }^{3}$, \\ Myung-Ki Shin ${ }^{4}$, Ved P. Kafle ${ }^{3}$, and Alex Galis ${ }^{5}$ \\ ${ }^{1}$ Hitachi, Japan \\ daisuke.matsubara.pj@hitachi.com \\ ${ }^{2}$ NEC, Japan \\ t-egawa@ct.jp.nec.com \\ ${ }^{3}$ NICT, Japan \\ \{nisinaga, kafle\}@nict.go.jp \\ ${ }^{4}$ ETRI, Korea \\ mkshin@etri.re.kr \\ ${ }^{5}$ University College London, United Kingdom \\ a.galis@ucl.ac.uk
}

\begin{abstract}
Advancements concerning research and development of Future Networks (FNs) technologies have been introduced in recent years, such as network virtualization and software defined/driven network (SDN), information centric networking (ICN), cloud networking, autonomic management, and open connectivity. In this context ITU-T has developed initial Recommendations that lay out the essential directions for subsequent detailed work including further standardization of Future Networks. This paper presents the background and the context of FNs' standardization, the results and future plans originated from the initial standardization work performed by ITU-T.
\end{abstract}

Keywords: Future Networks, Future Internet, standardization, ITU-T Y.3001.

\section{$1 \quad$ Introduction and Context}

In recent years the balance, evolution and relationships between various networking requirements have changed significantly creating the need for new networking systems. These changes include different new equipments and mobile devices connected to the public telecommunication network, significant number of data centers operating with the network representing the realization of cloud computing systems and large number of various sensors, actuators and other "things" connected in the network realizing Machine-to-Machine (M2M) and Internet of Things (IoT) services.

The research community and the telecom industry have made continuous efforts to investigate and develop Future Networks (FNs) technologies and systems. Various technologies such as network virtualization [10] and software defined networking (SDN) [14][17][19][21], cloud networking [13][18][20], information centric networking (ICN) [8], autonomic management [7][11][12], and open connectivity have been

\footnotetext{
* Invited Paper.
}

A. Galis and A. Gavras (Eds.): FIA 2013, LNCS 7858, pp. 27-38, 2013.

(C) The Author(s). This article is published with open access at link.springer.com 
examined and developed. Driven by this activity, International Telecommunication Union Telecommunication Standardization Sector (ITU-T) has started the standardization of FNs as networking systems to be deployed roughly in the 2015 - 2020 timeframe. FN standardization was initiated based on two commentary methods of analysis: top down method working from objectives and design goals of FNs, and bottom up method working from individual candidate technologies that are relatively mature.

The result of this analysis has been reflected in Recommendation ITU-T Y.3001 [1][16]. It includes also various candidate technologies as building blocks of FNs as such technologies tend to mature earlier than the overall architecture. For example, network virtualization technology, making use of Network Functions Virtualisation such as SDN [19][21], has already emerged. In addition, some technologies are being investigated in other standards development organizations (SDOs). It is important to understand and benefit from this ecosystem of technologies.

This paper describes the current ITU-T's accomplishments on FN standardization and its underlying concepts.

This paper is organized as follows. The next section provides an overview of FN standardization activities in ITU-T. Next the Recommendation ITU-T Y.3001 that describes the objectives and design goals of FNs is presented. Other FN-related Recommendations and the ITU-T future plan are also presented. A synthesis of related standardization and research activities is presented next. The final section provides concluding remarks.

\section{Future Network Standardization in ITU-T}

ITU-T Study Group 13 (SG13), a group for network architecture and the lead group for FN standardization in ITU-T, started its activities on FNs in early 2009. Since the discussion of FN was in its very early stage, ITU-T concluded that it is very important to listen to the voice of not only ITU-T members, but also experts including researchers outside of ITU-T. Consequently, Focus Group on Future Networks (FG-FN), a temporary organization open to all experts inside/outside of ITU-T, was established and its activity started in July 2009. It developed deliverables that later were transferred to SG13 and turned into Recommendations ITU-T Y.30xx series.

Recommendation ITU-T Y.3001 [1] - Future Networks: Objectives and Design Goals, describes four objectives and twelve design goals for FNs and as such it presents the first standard definition and description of FNs. The fundamental difference between FNs and other transport networks systems such as those using the Internet Protocol (IP) is the shift from a separate transport and service strata to a packet-based network with service and management-aware characteristics, which is based on shared (virtualized) combined processing, storage and communication / connectivity resources. As such FNs are aimed at a unified infrastructure which connects and orchestrate the future Internet of people, devices, content, clouds, and things.

In this Recommendation, four objectives were identified as essential concerns to which not enough attention was paid in designing current networks. They represent 
the differential characteristics of FNs when compared with the current networks. The four objectives identified and described in Y.3001 are service awareness, data awareness, environmental awareness, and social and economic awareness. Also twelve design goals were identified as advanced capabilities and features that are necessary for the realization of FNs. Fig. 1 shows the mapping of design goals to the objectives.

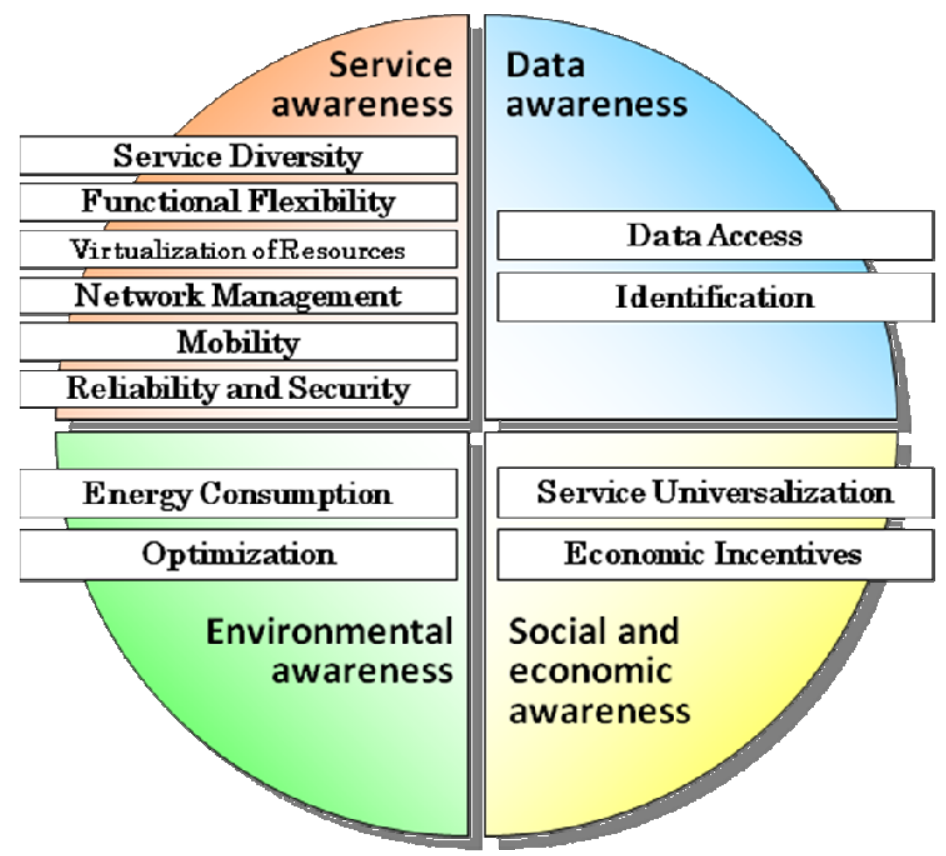

Fig. 1. Four objectives and twelve design goals of Future Networks [1]

\subsection{Service Awareness}

The number and range of services is expected to explode in the future. Today's network, whose basic design was introduced more than thirty years ago, has supported so far any service using its basic functionality and design. FNs are expected to support not only current services such as Email and web browsing, but also emerging services in an optimal way, by providing additional functionality and flexibility that can accommodate diverse and evolving service requirements.

FNs are aimed to support these services without drastic increases in, for instance, deployment and operational costs. In addition, FNs are required to be flexible and elastic so that they can adapt to new services. For example, if a service requires a certain process to be done inside the network, the network should provision dynamically all managed communication, computing, and storage resources that are needed for that service. Furthermore, these resources may be virtualized to allow flexible deployment and usage by the services. 
In order to support diverse services for mobile users including M2M communication devices, advanced mobility features that guarantee sufficient quality of service experience to the users in both homogeneous and heterogeneous mobile environments are needed.

Network and system management will play a significant role in allowing the network to take in and accommodate these diverse services. Network management will need to manage not only physical resources, but also virtual resources located inside the network. Unified management of FNs, which includes in-network autonomic management [7][11][12], is an approach where management and control functions are distributed and located or hosted in or close to the managed network and service elements, and the services itself may need to be managed along with the network in a unified manner.

Finally, the services will need to support the social infrastructure including mission critical services; hence FNs will require substantially enhanced security and reliability compared to the current networks.

An overview of service awareness in FNs is shown in Fig. 2.

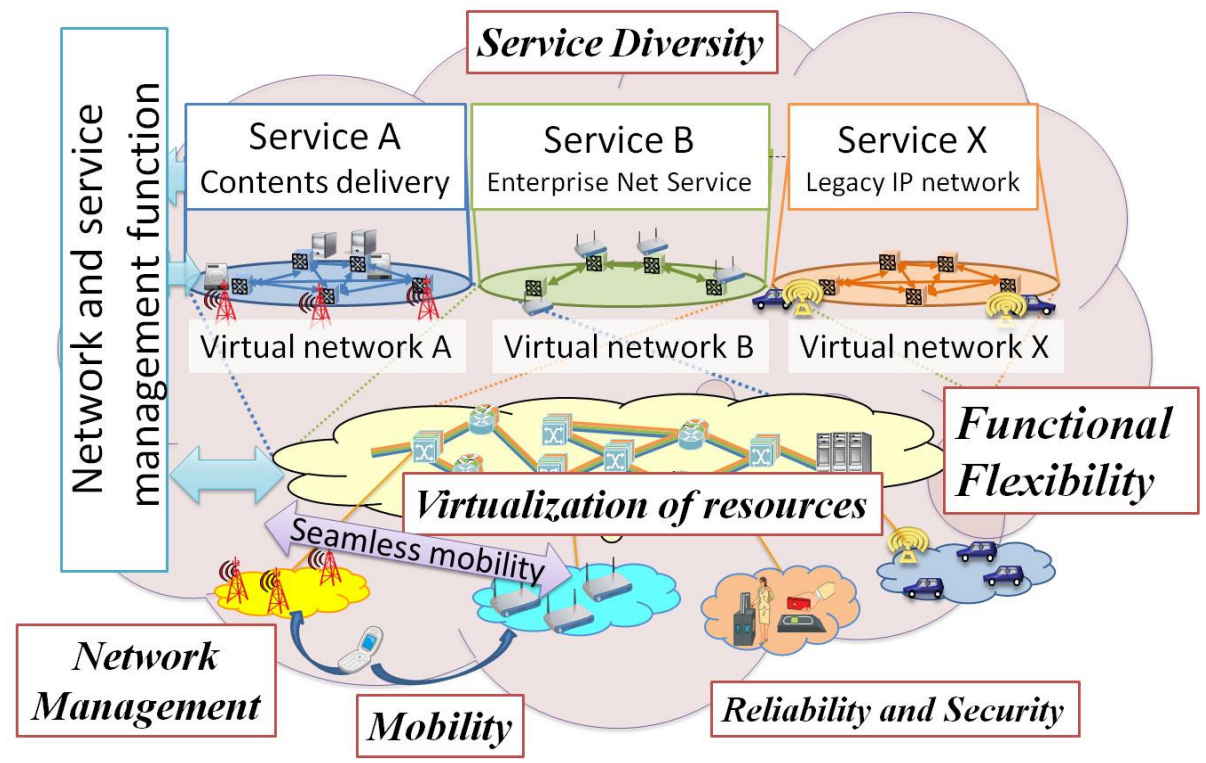

Fig. 2. Service awareness in FNs

A service-aware network is a rich landscape of network services, which can be discovered, negotiated, and contracted with by higher level services at the application level. These services need to be discoverable and describable by attributes such as capacity, throughput, QoS, latency, protocol support, availability, and security in a consistent format. They need to express cost and availability, scalability, and potentially elasticity and support for usage variations. They need to be supported by a negotiation service, which can implement contracts with consumers. FNs are necessary to accommodate these diverse services without a drastic increase in costs of deployment 
and operation. One method is to enable network operators to control their networks in a unified and programmable manner, and realize multiple isolated and flexible innetworks in order to support a broad range of network services that do not interfere with each other. From this viewpoint, promising technologies includes network virtualization [10][21] and SDN [14], and cloud networking [13][17][18][19][20] technologies.

ITU-T has successfully developed and published Recommendation ITU-T Y.3011 - Framework of Network Virtualization for FNs [2], which is the first Recommendation regarding service awareness in FNs from the perspective of ITU-T. Network virtualization is the process of combining hardware and software network resources and network functionality into a single, software-based administrative entity, a virtual network. Network virtualization involves platform virtualization, often combined with resource virtualization [21]. Network virtualization is also a method that allows multiple virtual networks called Logically Isolated Network Partitions (LINPs) to coexist in a single physical network. In Y.3011, the following eight design principles for realizing network virtualization are presented and investigated.

- Network abstraction: hiding the underlying characteristics of network resources and establishing simplified interfaces for accessing the network resources.

- Isolation: complete separation among the LINPs (e.g., from security and performance aspects).

- Topology awareness and quick reconfigurability: dynamic update of an LINP's capabilities without interruption of operations.

- Performance: avoidance of the performance degradation caused by the virtualization layer or adaptation layer.

- Programmability: programmable control plane and data plane so that users can use customized protocols, forwarding or routing functions in the LINP.

- Management: independent and unified management functions for each LINP.

- Mobility: movement support of virtual resources including users and services.

- Wireless: wireless characteristics support such as limited resource usage, and signal interference.

As the next step of Y.3011, more detailed requirements in realizing network virtualization are being envisaged in a separate Draft Recommendation ITU-T Y.FNvirtreq Requirements of Network Virtualization for Future Networks, which focuses on virtual resource management, service mobility, wireless virtualization, and candidate protocols and existing requirements for network virtualization.

In addition, SDN technologies are emerging and intensively discussed as one of solutions regarding network virtualization within telecom networks including mobile, data centers, and enterprise networks. Draft Recommendation ITU-T Y.FNsdn Framework of Telecom Software-Defined Networking (SDN), specifies requirements and use cases for SDN in telecom networks. SDN is defined as a new networking technology, which enables network operators to directly control and manage their networks and resources to best serve their customers' needs by writing simple programs, where controls and data forwarding are decoupled. Its properties include programmable controls, data forwarding abstraction, and virtualization support of the underlying networks infrastructure and resources. ITU-T is planning to collaborate on 
this topic with other SDOs such as Open Networking Foundation (ONF) and Internet Engineering Task Force (IETF).

\subsection{Data Awareness}

Current networks are mainly used for accessing and distributing information. To realize this, the networks establish a communication connection between an application process of each terminal (end-host) and exchange data using the connection. The exchange of information in current networks is based on the globally unique location IDs and location based routing as shown in Fig. 3 .

However, if identical information objects are placed in multiple locations as expected in FNs, it is not always optimal to access information using globally unique static location IDs. Identical contents may have the same content ID and the content can be accessed via a nearest cache using the content ID based routing as shown in Fig. 3. FNs are aimed at optimizing the handling of enormous amounts of data in a distributed environment with the users enabled to access desired data safely, easily, quickly, and accurately, regardless of their location. In the context of data awareness, "data" is not limited to specific data types such as audio or video contents, but includes all information that is accessible via the network.

In FNs, communication paradigms using IDs other than location IDs is envisaged. FNs aim to support communication using data (or content) IDs. Furthermore, it will support communication using node IDs, application process IDs, etc. These IDs need to be treated separately from location IDs and FNs should support not only separation of end point or node IDs and locators such as specified in Locator/ID Separation Protocol (LISP) and Recommendation ITU-T Y.2015, but also communication using data IDs, service IDs, etc.

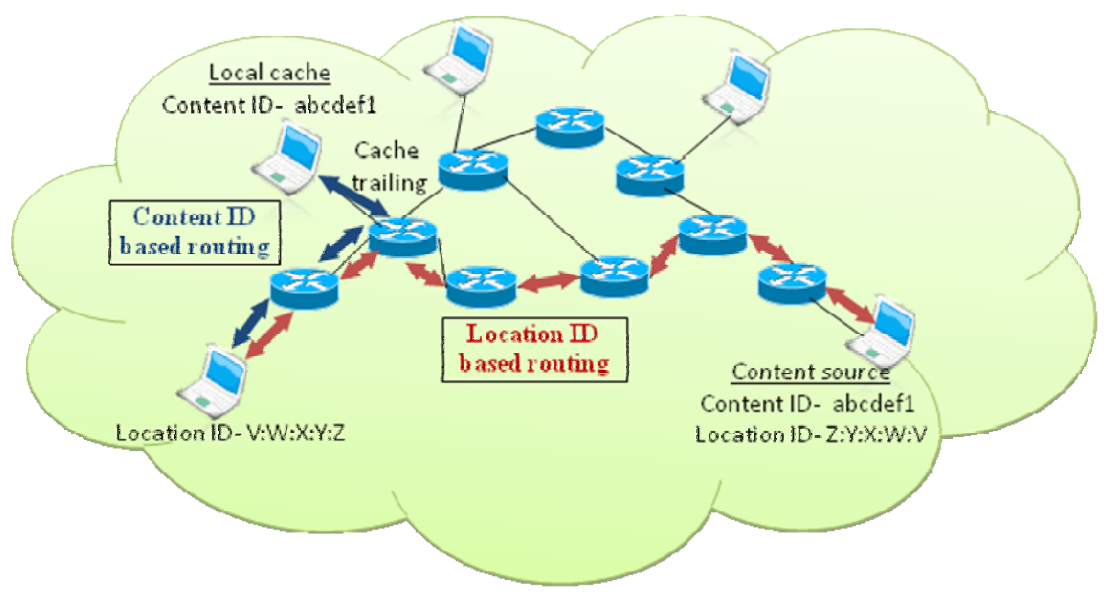

Fig. 3. Data awareness in FNs 
Recommendation ITU-T Y.3031 - Identification framework in future networks [4] is part of the series of FN-related Recommendations developed in ITU-T SG13. It complements the FN objectives and design goals specified in ITU-T Y.3001 by developing a new identification framework that would be helpful for intrinsic mobility support and optimal data access. It specifies the identification framework, after giving an analysis of identifiers being used in the current networks and their limitations. It mentions about the overloaded semantic of an IP address as an identifier, a locator and a forwarding tag, and consequent hindrances for mobility and multihoming services.

The identification framework is horizontally positioned between the communication objects (such as user, device, data, and service) and physical networks forwarding data from one place to another. The framework consists of four components: ID discovery service, ID space, ID mapping registry, and ID mapping service. The ID discovery service discovers various types of IDs related to communication objects. The ID space defines and manages various kinds of IDs (e.g., user IDs, data or content IDs, service IDs, node IDs, and location IDs). The ID mapping registry maintains mapping relationships between various types of IDs. The ID mapping service performs mappings of IDs of one type with the IDs of other types. The ID mapping service utilizes the ID mappings obtained from the ID mapping registry to achieve seamless services over heterogeneous physical networks, such as IPv6, IP IPv4, or non-IP networks that may use different protocols and media for forwarding data.

ITU-T is currently working on Y.FNDAN - Framework of Data Aware Networking for Future Networks, which gives an overview of Data Aware Networks (DAN). DAN is a technology, which optimizes handling of enormous amount of data in a distributed environment and enables users to access desired data safely, easily, quickly, and accurately, regardless of their location. In addition, due to the awareness feature of this technology, it enables networks to understand users' requests and to react accordingly in order to support adaptive data dissemination.

The essence of DAN lies in the name-based routing in which the data or the request for the data is routed inside the network not by its location but by its name or ID (i.e., routing and forwarding is based on data ID). It captures many aspects of ongoing research works such as content-centric networking (CCN) [5] and information-centric networking (ICN) [8]. Y.FNDAN will provide general properties and high-level requirements of DAN such as naming, routing, in-network caching, in-network processing, and data security.

\subsection{Environmental Awareness}

The ratio of carbon dioxide (CO2) that information and communication technology (ICT) industry produces is two percent of the entire CO2 emission [15]. This includes $\mathrm{CO} 2$ contribution by PCs, servers, cooling systems, fixed and mobile telephony, local area networks (LANs), office telecommunications and printers.

Internet traffic is growing year by year. It has been predicted that the traffic triples every five years period and will reach 1.3 zettabytes by 2016. Considering the necessity to transmit information via the network, the increase in traffic will mean ever 
increase in energy consumption; hence the emission of $\mathrm{CO} 2$ will most likely continue to increase. For this reason, FNs aims at minimizing and managing the energy needed to transmit bits in the device-level, equipment-level, and system-level. At the same time, energy can be managed in a better manner by utilizing ICT for various industries such as manufacturing and distribution of goods.

Recommendation ITU-T Y.3021 - Framework of Energy Saving for Future Networks [5] reviews various energy saving technologies and categorizes them into two according to the basic strategy. One is to reduce the network capacity by reducing traffic (e.g., by caching) or peak load shift. The other is improvement of energy efficiency by dynamic control (e.g., clock gating, sleep mode control, etc.) or less power (e.g., LSI fabrication, thermal design, etc.) Then it describes a feedback loop among measurement, control and management as the framework of energy saving.

Also, each ITU-T Recommendation related to FNs has an environmental consideration section that assesses environmental impact of the technology. This is inspired by security consideration section commonly addressed in ICT standards.

There are large numbers of standardization activities that contribute to realize environmental objective of Y.3001. Within ITU-T activities there are Recommendations that defines a power charger specification for mobile terminals to reduce e-waste, assessment methodology for environmental impact of ICT, etc. Many of them are applicable to FNs.

\subsection{Social and Economic Awareness}

As networks are evolving from just connecting people with common interest to a social infrastructure, service universalization is becoming a key objective in realizing the new networking. The right to have access to a global network will be one of the fundamental rights in the future, and the right should not be limited based on the location of the individual user.

Telecommunication networks have become an essential infrastructure utility that is indispensable to our society, much similar to electricity, gas, and water. For this reason, FNs aims to take into consideration the social and economical aspects when realizing the architecture.

It is also necessary for the network to evolve in a sound and consistent manner. Public networks such as telephony networks have been invested and operated mainly by government-owned companies and have supported and fostered the national industry. Recently private investment has become active and capital market has been introduced in investment and operation of network infrastructure. At the same time, the relationship between the investment model and the profit distribution model has been distorted and it is becoming an obstructive factor for the appropriate development of the market.

FNs should take explicitly into consideration the lowering of the barriers for stakeholders to enter the market and providing a sustainable competitive environment.

Network architecture indirectly but undoubtedly affects society and business by providing the playground for social activity and business. ITU-T Y.3001 is emphasizing that FNs should consider social and economic issues such as the barrier to enter 
the market or the lifecycle cost for deployment and sustainability, although Y.3001 focuses manly on technical aspect. This is an interdisciplinary issue between technology and policy, which should not be decided by standards, but by the market through competition.

ITU-T started a socio-economic analysis to from a framework called Draft Recommendation ITU-T Y.FNsocioeconomic - Socio-Economic Aware Design of Future Network Technology. Current draft provides a framework to anticipate the socioeconomic impact of the technology at technology design time. When a candidate FNs technology is provided, it recommends to take into account the relevant set of stakeholders, tussles emerging among them, and the range of available choices, to anticipate either a stable and incentives-compatible or an unstable outcome resulting from deploying the technology, to identify potential spillover (unwanted) effects from the technology's primary functionality to another functionality, and to help design a technology for FNs that is in-line with the respective socio-economic design goals and objectives.

\section{Future Plan}

Standardization activities of FNs are gaining momentum. For example, SDN that is closely related to the service-awareness objective is becoming a hot topic in ICT industry. ITU-T SG 13 therefore decided to divide the group involved in standardization of FNs into three groups, first group for service awareness including SDN, second group for data awareness, and third group for environment and socio-economic awareness and short-term realization of FNs. FNs are a huge target, and various areas needs to be discussed for future standardization apart from the draft Recommendations mentioned in the previous sections. One of the most important area is unified management of FNs, which includes in-network autonomic management [7][11][12]. The benefits are the inherent support for self-management features, higher automation and autonomicity capabilities, easier use of management tools and empowering the network with inbuilt cognition and intelligence. Additional benefits include reduction and optimization in the amount of external management interactions, which is a key to the minimization of manual interaction and the sustaining of manageability of large networked systems and moving from a managed object paradigm to one of management by objectives.

The three groups in ITU-T SG 13 are far from enough to cover all these aspects of FNs. And there are many existing and ongoing work in other ITU-T Study Groups and other SDOs. ITU-T will be seeking ways to collaborate with other SDOs considering the technology research and market needs, which is essential task for realizing FNs.

\section{$4 \quad$ Related Research and Standardization and Programs}

Fig. 4 shows a chronology of FN related research and development activity along with ITU-T standardization activities for FNs. The NewArch project initiated in 2000 
by several US universities and institutes is the ancestor of Future Internet architecture design projects advocating for "clean slate" design approach. It was founded by DARPA (Defense Advanced Research Projects Agency), which was the funding body supporting the initial design of the Internet. The objective of this project was to define a network architecture as "advanced design principles for making use of protocols and algorithms." The 100x100 Clean Slate Project (2000 - 2005) was an NSF (National Science Foundation) - supported collaborative project launched and its slogan was "100Mbps connectivity to 100 million homes" with new technology. The FIND (Future Internet Design) and FIA (Future Internet Architecture) are also funded by NSF. The FIND was a long-term initiative of the NSF NeTS research program and it also focused to the "clean slate" design approach. More than 40 projects were established and four large projects (FIA projects) are generated as the result of FIND. For testing brand new network architecture design through above projects, GENI (Global Environment for Network Innovations) was initiated in 2005 by NSF.

In EU, more than 150 projects clustered as EU Future Internet Assembly (FIA) [6][9] are developing networking systems for the Future Internet.

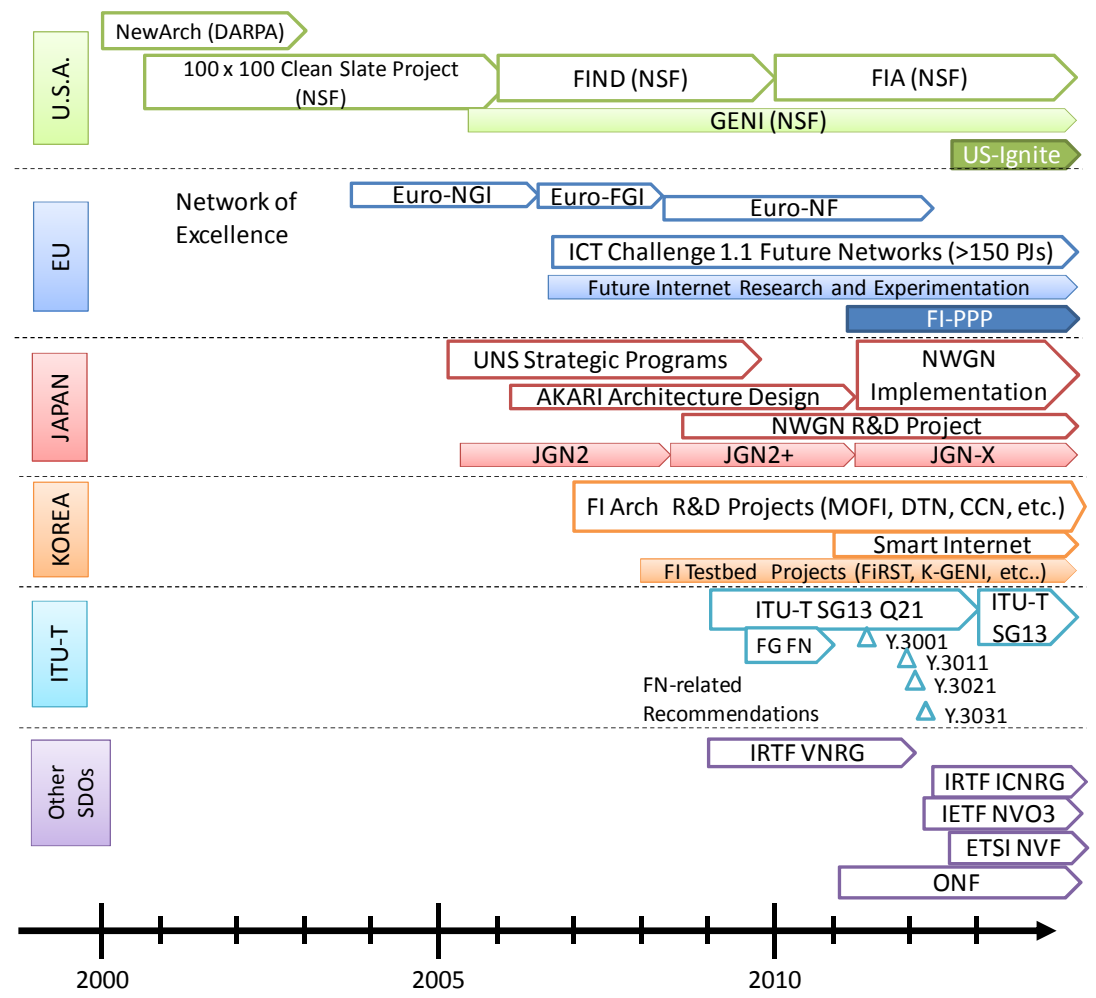

Fig. 4. Standardization and Research activities regarding FNs 
Japanese government announced UNS (Ubiquitous Network Society) strategy program in 2005 and AKARI project was initiated in 2006 to design a NeW Generation Network (NWGN) architecture by NICT. It is continuing as one project in NWGN R\&D Project. New Generation Network Test beds JGN-X is now under operating with the network virtualization technology.

In Korea, FIArch projects such as Mobile Oriented Future Internet (MOFI), Delay Tolerant Networking (DTN), and CCN were launched in 2007. Future Internet Research for Sustainable Testbed (FiRST) and international federation projects such as K-GENI were initiated in 2008. Korean government has also announced Smart Internet as a first deployment model of Future Internet in 2011.

There are also related standardization activities in other SDOs such as ETSI Network Functions Virtualization (NFV), Open Networking Foundation (ONF), IETF Network Virtualization Overlays (NVO3), Internet Research Task Force (IRTF) Virtual Networks Research Group (VNRG), and IRTF Information-Centric Networking Research Group (ICNRG).

\section{$5 \quad$ Concluding Remarks}

ITU-T has developed and published during 2009 - 2012 four important Recommendations: Y.3001, Y.3011, Y.3021 and Y.3031, representing the first standard descriptions of Future Networks. In addition to connectivity services, FNs are characterized by four objectives and twelve design goals. These design goals are advanced capabilities, features, and new network services that are needed together in the realization of FNs. As the standardization work progressed through discussions with various experts in this field, ITU-T was able to capture and identify the key characteristics and important aspects of FNs and specify them in these documents. We believe that these Recommendations will provide sound foundation and appropriate guidance for subsequent FNs realization, standardization, research and development.

Acknowledgment. The authors would like to thank Sangjin Jeong, Hideki Otsuki, Toshihiko Kurita, Martin Waldburger, Alojz Hudobivnik, Naotaka Morita, HyoungJun Kim, and Chaesub Lee for their work and contributions to the ITU-T FN activities. This article was partially supported by the European Union UniverSELF project, National Institute of Information and Communications Technology (NICT), and the ICT Standardization program of Korea Communications Commission (KCC).

Open Access. This article is distributed under the terms of the Creative Commons Attribution Noncommercial License which permits any noncommercial use, distribution, and reproduction in any medium, provided the original author(s) and source are credited.

\section{References}

1. Recommendation ITU-T Y.3001, Future Network Vision - Objectives and Design Goals (2011)

2. Recommendation ITU-T Y.3011, Framework of Network Virtualization for Future Networks (2012) 
3. Recommendation ITU-T Y.3021, Framework of energy saving for Future Networks (2012)

4. Recommendation ITU-T Y.3031, Identification Framework in Future Networks (2012)

5. Jacobson, V., Smetters, D.K., Thornton, J.D., Plass, M.F., Briggs, N.H., Braynard, R.L.: Networking named content. In: CoNEXT 2009, Rome (December 2009)

6. Álvarez, F.A., et al.: FIA 2012. LNCS, vol. 7281, pp. 1-252. Springer, Heidelberg (2012)

7. Clayman, S., Clegg, R., Mamatas, L., Pavlou, G., Galis, A.: Monitoring, aggregation and filtering for efficient management of virtual networks. In: IEEE International Conference on Network and Service Management (CNSM) (October 2011)

8. Kutscher, D., Ahlgren, B., Kar, H., Ohlman, B., Oueslati, S., Solis, I.: Information-centric networking. Dagstuhl Seminar (2010)

9. Domingue, J., et al.: FIA 2011. LNCS, vol. 6656, pp. 1-465. Springer, Heidelberg (2011)

10. Galis, A., Denazis, S., Brou, C., Klein, C. (eds.): Programmable networks for IP service deployment, pp. 1-450. Artech House Books (June 2004) ISBN: 1-58053-745-6

11. Clegg, R.G., Clayman, S., Pavlou, G., Mamatas, L., Galis, A.: On the selection of management and monitoring nodes in dynamic networks. IEEE Transactions on Computers (99), 1-15 (2012)

12. Ciavaglia, L., et al.: Realizing autonomics for future networks. In: Future Network and Mobile Summit, Warsaw, Poland (June 2011)

13. Rochwerger, B., et al.: The RESERVOIR model and architecture for open federated cloud computing. IBM System Journal, Special Edition on Internet Scale Data Centers 53(4) (2009)

14. Rubio-Loyola, J., et al.: Scalable service deployment on software defined networks. IEEE Communications Magazine 49(12), 84-93 (2011)

15. http://www.gartner.com/it/page.jsp?id=503867 (visited on December 14, 2012)

16. Matsubara, D., Egawa, T., Nishinaga, N., Shin, M.K., Kafle, V.P., Galis, A.: Towards Future Networks: a viewpoint from ITU-T. IEEE Communication Magazine - Special Issue on Telecommunications Standards (to be published in Q2 2013), http: / /www. comsoc.org/files/Publications/Magazines/ci/cfp/cf pcommag 0313. html

17. Software-Defined Networking - Open Networking Foundation (ONF) (April 2012), http: / /www. opennetworking.org/images/stories/downloads/white -papers/wp-sdn-newnorm.pdf

18. Openstack - quantum wiki, http://wiki.openstack.org/Quantum

19. SDN Document Library, http://www.sdncentral.com/sdn-documentlibrary/

20. Chapman, C., Emmerich, E., Marquez, F.G., Clayman, S., Galis, A.: Software Architecture Definition for On-demand Cloud Provisioning. Springer Journal on Cluster Computing (May 2011), http: / /www. springerlink. com/content/m31np5112525167v/, doi:10.1007/s10586-011-0152-0

21. “Network Functions Virtualisation" ETSI White Paper (October 2012), http://portal.etsi.org/NFV/NFV_White_Paper.pdf 